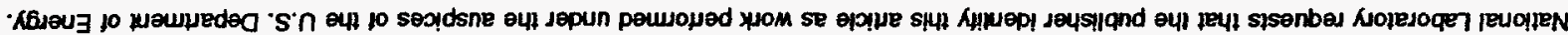

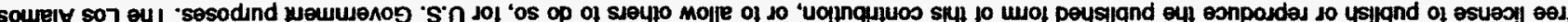

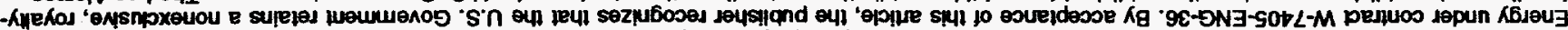

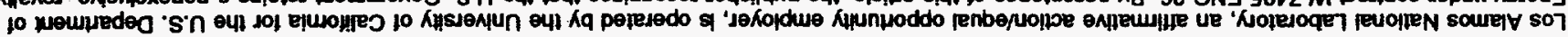

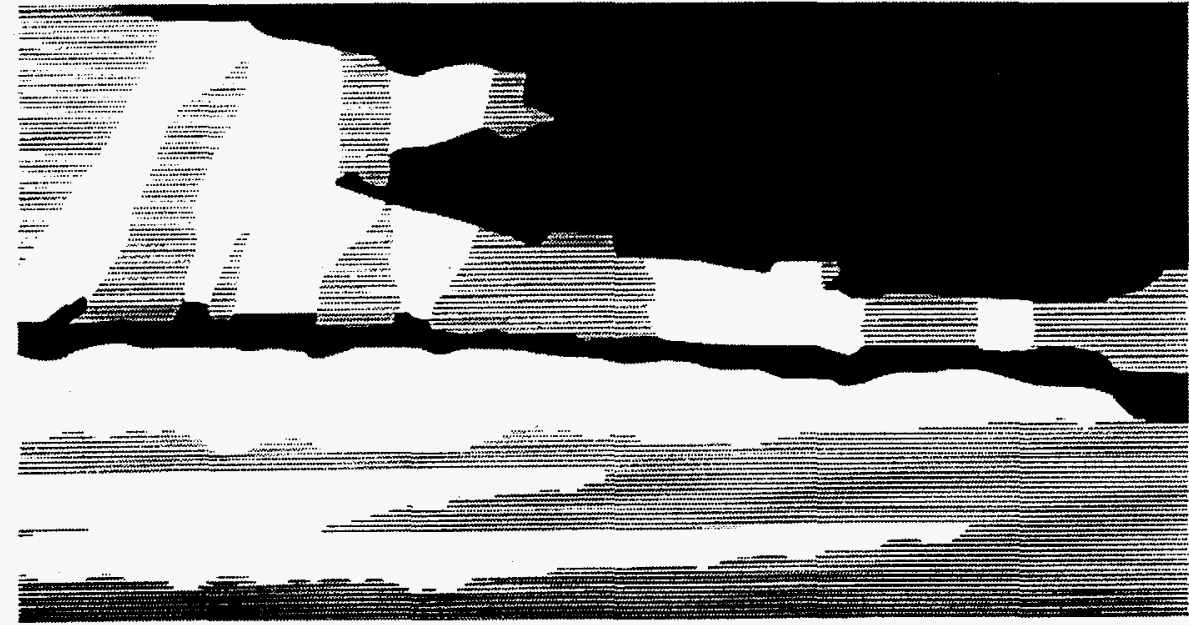

$$
\begin{aligned}
& \text { (IISO) uo!jeusojul }
\end{aligned}
$$

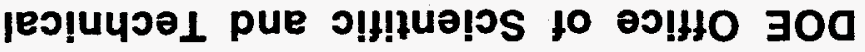

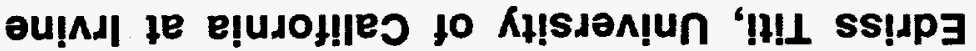

$$
\begin{aligned}
& \text { zO!Mә!)גejous d!!Od } \\
& \text { ddפ। 'olod maspu } \\
& \text { ddפ। 'eb!pen - } g \\
& \text { uOpsaew 'r } \\
& \text { WHX 'u!ןobdew uә7 }
\end{aligned}
$$

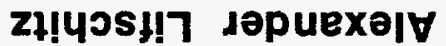

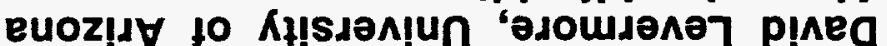

$$
\begin{aligned}
& \text { ddפI 'souor pieuog } \\
& \text { u.nqyoos } \cdot \mathrm{g} \\
& \text { L-1 '!०पว 6UnOKOOM } \\
& L-1 \text { 'esseureo ofjəqoy } \\
& \Lambda_{1} \hat{e} \text { eg } \cdot g \\
& \text { jeqi७ Yjew } \\
& L-\perp \text { 'wןOH INJdea }
\end{aligned}
$$

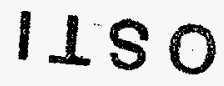

Бu!ןpow so!ueuka ueəso әןeososaw

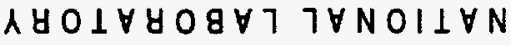 somej $\mathbf{s 0 7}$}

:on pantuans

:(s)romn

:əเ!L 


\title{
Mesoscale Ocean Dynamics Modeling
}

Darryl Holm*, Mark Alber, B. Bayly, Roberto Camassa, Wooyoung Choi, B. Cockburn, Donald Jones, David Levermore (University of Arizona), Alexander Lifschitz, Len Margolin,

J. Marsden, B. Nadiga, Andrew Poje, Poitr Smolarkiewicz, and Edriss Titi (UC Irvine)

\begin{abstract}
This is the final report of a three-year, Laboratory-Directed Research and Development (LDRD) project at the Los Alamos National Laboratory (LANL). The ocean is a very complex nonlinear system that exhibits turbulence on essentially all scales, multiple equilibria, and significant intrinsic variability. Modeling the ocean's dynamics at mesoscales is of fundamental importance for long-time-scale climate predictions. A major goal of this project has been to coordinate, strengthen, and focus the efforts of applied mathematicians, computer scientists, computational physicists and engineers (at LANL and a consortium of Universities) in a joint effort addressing the issues in mesoscale ocean dynamics. The project combines expertise in the core competencies of high performance computing and theory of complex systems in a new way that has great potential for improving ocean models now running on the Connection Machines CM-200 and CM-5 and on the Cray T3D.
\end{abstract}

\section{Background and Research Objectives}

Modeling the ocean's dynamics at mesoscales ( $>20 \mathrm{~km}$, the peak of the kinetic energy spectrum) is of fundamental importance for long-time-scale climate predictions. This is because the ocean responds to forcing agents on a much longer time scale than the atmosphere does (essentially due to the much bigger density and specific heat of water relative to air), so that much of the long-time-scale driving of the atmosphere can be attributed to the ocean. This is already evident from climate effects due to oceanic events at even relatively short time scales, such as El Nino. Thus, a thorough understanding of ocean dynamics is particularly crucial for investigating long term phenomena like global warming.

The ocean is a very complex nonlinear system. It is turbulent on essentially all scales, exhibits multiple equilibria, and has intrinsic variability on time scales ranging from a few years

*Principal investigator, e-mail: dholm @lanl.gov 
to thousands of years. There is a correspondingly wide range of spatial scales in the ocean, most of which is unresolved in global modeling of ocean dynamics with supercomputers. The current state of supercomputer speeds and memories allow one to compute global circulation on meshes with horizontal dimensions of about $55 \mathrm{~km}$. However, measurements of the kinetic energy spectrum of the ocean show that most of the energy resides in scales of $20 \mathrm{~km}$ or less. That is, most of the kinetic energy of the ocean is found in small eddies that are not resolved on the finest meshes possible today. The effects of this energy at small scales on the dynamics at mesoscales and larger must be modeled computationally. It is difficult to foresee all the relevant phenomena that can occur in mesoscale ocean dynamics, much less to understand their relative importance, without a solid mathematical analysis of the underlying equations. The objective of the analysis is to obtain a full understanding of the nonlinear phenomena contained in the ocean dynamics equations at the most fundamental mathematical level consistent with the numerical modeling. This understanding can then be used to spark new approaches to solving practical engineering and scientific problems, as well as to designing more effective computer simulations and suggesting new directions for experimental investigations.

The overall goal of the project has been to help develop effective mathematical and computational tools for analyzing the nonlinear behavior appearing in mesoscale ocean dynamics, in the framework of the Laboratory's Center for Nonlinear Studies (CNLS) unique and special connections with the world community in nonlinear science. The primary emphasis of the work has been on the development of a combined analytical and numerical understanding that complements and supports the development and interpretation of ongoing experimental and computational efforts both at the Laboratory and in the academic community. Thus, our goal is to help coordinate a nonlinear science framework at the CNLS for investigating mesoscale ocean dynamic flows in strong scientific collaboration with the external nonlinear science and oceanographic community.

\section{Importance to LANL's Science and Technology Base and National R\&D Needs}

This research combines two of the Laboratory's core competencies in a new way. These are high performance computing and theory of complex systems. At Los Alamos there is presently a concerted effort to develop the world's best capability in global ocean dynamics computations on the Connection Machine. This "Grand Challenge" effort involves personnel in the Advanced Computing Laboratory, Theoretical Division, Physics Division, Earth and Environmental Sciences Division, the Institute for Geophysics and Planetary Physics (IGPP), and the CNLS. 
The ocean's dynamics is determined by the combined effects of nonlinearity, stratification, dispersion, rotation, topographical and boundary forcing, and turbulence. This is a major open problem, with many potentially important applications and potential implications for national and global needs. Our approach established a framework for investigating ocean dynamic flows from the viewpoint of nonlinear science and dynamical systems methods, and an external collaboration network for further developing the applications of this understanding. Moreover, we are working within the computational science environment of the Laboratory, as well as attracting collaborators from the national ocean modeling community.

A significant benefit to the Laboratory is the number and quality of postdoctoral fellows brought to Los Alamos through this type of research collaboration.

\section{Scientific Approach and Results}

Understanding global climate and climate change is one of the grand challenge problems. For reasons described in the previous section, a model that correctly reproduces the global circulation of the ocean at mesoscales and larger, and that can generate intrinsic variability for physically correct time scales, is essential in understanding global climate and climate change. However, present day global ocean models are built on numerical methods that are nearly twenty years old, and that are more appropriate to deterministic flows. Furthermore, we are currently limited (by the capabilities of today's supercomputers) to meshes that do not resolve most of the energy scales of the problems. In our workshops and collaborations with visitors in this work, we are helping to address the questions that will allow genuine collaboration between the ocean modelers, the mathematicians and the nonlinear scientists.

In the workshops, the ocean modelers focused on the state of the art in global ocean modeling, and what they perceived as the current needs of the models in terms of physics, numerics, and computer resources. Since predictability is a complex and largely undefined subject, they focused on efforts to model the global thermohaline circulation patterns. There is general agreement that these circulations, and in particular the so-called conveyor belt, represent important aspects of the global climate system.

The mathematicians focused on current research in the integration of nonlinear partial differential equations over long times. Their attention centered on the ideas of the "slow manifold," proposed by Leith and Lorenz within the meteorological community more than twenty years ago, and the "inertial manifold," a relatively new idea that until recently has been entirely the province of the mathematician. New Approximate Inertial Manifold (AIM) techniques for finite-difference simulations of ocean dynamics are one of the emerging results 
from these discussions and scientific collaborations in this project. By optimizing the use of information at spatial grid points to capture the dominant degrees of freedom, these AIM techniques hold great promise for producing more accurate ocean modeling without substantial increase in the computer time.

Over its three years duration, the project funded studies centered around major workshops in: foundations of balanced dynamics for mesoscale ocean modeling, geostrophic turbulence, approximate inertial manifolds for finite difference methods, applications of proper orthogonal decomposition, predictability in ocean modeling, asymptotic behavior of nonlinear shallow water equations, and modeling turbulent flows. Our primary purpose in each study was to bring together practicing ocean modelers and applied mathematicians interested in solving nonlinear partial differential equations. The workshops were held in Los Alamos, and most were jointly sponsored by the IGPP and by the CNLS. One day of the 1993 CNLS Annual Conference, titled "Modeling Forces of Nature," was devoted to discussions of mesoscale ocean dynamics, including a discussion of hurricane dynamics. In 1995, the CNLS Annual Conference, titled "Nonlinear Phenomena in Ocean Dynamics," was entirely devoted to ocean dynamics. The Proceedings of the first conference has already appeared as a special volume of the refereed journal, Physica D, and that for the second will appear in 1996.

We have derived new equations describing the time asymptotic effects of the nonlinear dispersion present due to hydrostatic pressure imbalance in the vertically averaged Euler equations for shallow, free-surface, incompressible hydrodynamics. For this, we used multiple time-scale asymptotic expansions that included full topography and boundary information. In the course of this work we have developed a new method of "Hamiltonian asymptotics." This new method produces approximate descriptions of fluid dynamics that retain the Hamiltonian properties of the exact original system at each order in the asymptotic expansion. (This implies that the approximate equations conserve energy and potential vorticity at each order.)

We have obtained approximate descriptions of fluid dynamics at two successive orders in an asymptotic expansion in the small aspect ratio of the flow that retain the Hamiltonian properties of the original system at both levels of approximation. The conservation laws implied by the Hamiltonian nature of these equations at each order in the expansion led to an analytical estimate of the rate at which the solution at higher order diverges from the leading order solution due to nonlinear dispersion. In the last year of the project, the method of Hamiltonian asymptotics continued to produce new scientific results, as we derived new Hamiltonian balance equations and new equations for wave mean-flow interaction dynamics between internal waves and mean ocean currents. These results will be published in the Physica D Proceedings volume for the 1995 CNLS Annual Conference. 


\section{Publications}

1. Alber, M., Camassa, R., Holm, D. D., and Marsden, J., "The Geometry of Weak Solutions of Certain Integrable Nonlinear PDEs," in NEEDS '94 Proceedings, V. G. Makhankov, A. R. Bishop and D. D. Holm, ed., World Scientific, Singapore, pp 3-8 (1995).

2. Alber, M., Camassa, R., Holm, D. D., and Marsden, J., "On the Link between Umbilic Geodesics and Soliton Solutions of Nonlinear PDEs," Proc. Roy. Soc., 450, 677-692 (1995).

3. Alber, M., Camassa, R., Holm, D. D., and Marsden, J., "The Geometry of Peaked Solitons and Billiard Solutions of a Class of Integrable PDE's," Lett. Math. Phys., 32, 137--151 (1994).

4. Bayly, B., Holm, D. D., and Lifschitz, A., "Three-Dimensional Stability of Elliptical Vortex Columns in External Strain Flows," Trans. Roy. Soc. London, to appear (1996).

5. Camassa, R.and Holm, D. D., "An Integrable Shallow Water Equation with Peaked Solitons," Phys. Rev. Lett., 71, 1661-1664 (1993).

6. Camassa, R., Holm, D. D., and Hyman, J.M., "A New Integrable Shallow Water Equation," Adv. Appl. Mech., 31, pp 1--33 (1994).

7. Camassa, R., Holm, D. D., and Levermore, C. D., "Long-Time Effects of Bottom Topography in Shallow Water," Physica D, to appear (1996).

8. Choi, W., "Nonlinear Evolution Equations for Two-Dimensional Surface Waves in a Fluid of Finite Depth," Journal of Fluid Mechanics, 295, 381-394 (1995).

9. Choi, W. and Camassa, R., "Weakly Nonlinear Internal Waves in a Two-Fluid System," LANL report LAUR-95-2996 (1995).

10. Allen, J. and Holm, D. D., "Hamilton's Principle for Shallow Water Models with Height as the Independent Variable," Physica D, to appear (1996).

11. Gjaja, I. and Holm, D. D., "Self-Consistent Wave-Mean Flow Interaction Dynamics and its Hamiltonian Formulation for a Rotating Stratified Incompressible Fluid," Physica D, to appear (1996).

12. Holm, D. D., "Hamiltonian Balance Equations," Physica D, to appear (1996).

13. Holm, D. D., "The Ideal Craik-Leibovich Equations," Physica D, to appear (1996).

14. Jones, D., Titi, E., and Cockburn, B., "Estimating the Number of Asymptotic Degrees of Freedom for Noninear Dissipative Systems," Mathematics of Computations, to appear (1996).

15. Jones, D., Titi, E., and Cockburn, B., "Determining Degrees of Freedom for Nonlinear Dissipative Equations," C.R. Acad. Sci Paris Sér I, 321, 563-568 (1995). 
16. Jones, D., "On the Behavior of Attractors Under Finite Difference Approximations," Num. Funt. Anal. and Opt., 17 (1996).

17. Jones, D., Poje, A., and Margolin, L., "Nonlinear Difference Approximations for Evolutionary PDEs, in NEEDS '94 Proceedings, V. G. Makhankov, A. R. Bishop and D. D. Holm, ed., World Scientific, Singapore, pp 65-75 (1995).

18. Jones, D., Poje, A., and Margolin, L., "Enslaved Finite Difference Schemes for Nonlinear Dissipative PDEs," Num. Meth. for PDEs, 12, 13-40 (1996).

19. Jones, D. and Titi, E., " $\mathrm{C}^{1}$ Approximations of Inertial Manifolds for Dissipative Nonlinear Equations,' Journal of Differential Equations, to appear (1996).

20. Nadiga, B., Margolin, L., and Smolarkiewicz, P., "Semi-Langrangian Shallow Water Modeling on the CM-5," Parallel Computational Fluid Dynamics, Elsevier Science, pp 529-536 (1996).

21. Nadiga, B., "An Adaptive Discrete Velocity Model for the Shallow Water Equations," Journal of Computational Physics, 121, pp 271-280 (1995).

22. Nadiga, B., Margolin, L., and Smolarkiewicz, P., "Dispersive Shallow Fluid Flow over a Mountain," CNLS Newsletter (1995).

23. Nadiga, B., Margolin, L., and Smolarkiewicz, P., "Various Approximations of the Shallow Fluid Flow over an Obstacle," submitted to Physics of Fluids (1995). 


\section{DISCLAIMER}

This report was prepared as an account of work sponsored by an agency of the United States Government. Neither the United States Government nor any agency thereof, nor any of their employees, makes any warranty, express or implied, or assumes any legal liability or responsibility for the accuracy, completeness, or usefulness of any information, apparatus, product, or process disclosed, or represents that its use would not infringe privately owned rights. Reference herein to any specific commercial product, process, or service by trade name, trademark, manufacturer, or otherwise does not necessarily constitute or imply its endorsement, recommendation, or favoring by the United States Government or any agency thereof. The views and opinions of authors expressed herein do not necessarily state or reflect those of the United States Government or any agency thereof. 


\section{DISCLAIMER}

This report was prepared as an account of work sponsored by an agency of the United States Government. Neither the United States Government nor any agency thereof, nor any of their employees, makes any warranty, express or implied, or assumes any legal liability or responsibility for the accuracy, completeness, or usefulness of any information, apparatus, product, or process disclosed, or represents that its use would not infringe privately owned rights. Reference herein to any specific commercial product, process, or service by trade name, trademark, manufacturer, or otherwise does not necessarily constitute or imply its endorsement, recommendation, or favoring by the United States Government or any agency thereof. The views and opinions of authors expressed herein do not necessarily state or reflect those of the United States Government or any agency thereof. 